La VC suele localizarse en los miembros inferiores como púrpura palpable y el hallazgo histológico más común es la vasculitis leucocitoclástica con IFD positiva para $\mathrm{IgG}$, $\mathrm{IgM}$ y $\mathrm{C} 3^{7}$.

El tratamiento de la VC dependerá de la etiopatogenia y la severidad del cuadro clínico. En los casos leves a moderados asociados a SSp, consiste en evitar las bajas temperaturas, reposo y fármacos como antiinflamatorios no esteroides, colchicina o dapsona.

\section{BIBLIOGRAFÍA}

1. Ramos-Casals $M$, Stone $J H$, Cid MC, Bosch $X$. The cryoglobulinaemias. Lancet 2012;379(9813):348-360.

2. Brouet JC, Clauvel JP, Danon F, Klein M, et ál. Biologic and clinical significance of cryoglobulins. A report of 86 cases. Am J Med 1974;57:775-788.

3. Cacoub P, Comarmond C, Domont F, Savey L, et ál. Cryoglobulinemia vasculitis. Am J Med 2015;128:950-955.

4. Terrier B, Cacoub P. Cryoglobulinemia vasculitis: an update. Curr Opin Rheumatol 2013;25:10-18.

5. Ferri C, Sebastiani M, Giuggioli D, Cazzato M, et ál. Mixed cryoglobulinemia: demographic, clinical, and serologic features and survival in 231 patients. Semin Arthritis Rheum 2004;33:355-374.

6. Baldini C, Pepe P, Quartuccio L, Priori R, et ál. Primary Sjogren's syndrome as a multi-organ disease: impact of the
En casos graves con compromiso sistémico, se utilizan corticosteroides; inmunosupresores como micofenolato, azatioprina y ciclofosfamida; rituximab, o plasmaféresis ${ }^{4,9}$.

Destacamos el rol del médico dermatólogo en el diagnóstico precoz de una enfermedad sistémica a través de sus manifestaciones cutáneas. Por otro lado, resaltamos el manejo multidisciplinario en el tratamiento y el seguimiento de estos pacientes.

serological profile on the clinical presentation of the disease in a large cohort of Italian patients. Rheumatology (Oxford) 2014;53:839-844.

7. Singh AG, Singh S, Matteson EL. Rate, risk factors and causes of mortality in patients with Sjögren's syndrome: a systematic review and meta-analysis of cohort studies. Rheumatology (Oxford) 2016;55:450-460.

8. Retamozo S, Gheitasi L, Quartuccio L, Kostov B, et ál. Cryoglobulinaemic vasculitis at diagnosis predicts mortality in primary Sjögren syndrome: analysis of 515 patients. Rheumatology 2016;55:1443-1451.

9. Silva F, Pinto C, Barbosa A, Borges T, et ál. New insights in cryoglobulinemic vasculitis. J Autoimmun 2019;105:102313.

\title{
DERMATÓLOGOS JÓVENES
}

\section{^ Elección múltiple: LESIONES PURPÚRICAS EN LOS MIEMBROS INFERIORES}

\section{Paula Johana Barba, Pilar Targize Vaquero y Florencia Guillermina Martínez \\ Servicio de Dermatología, HIGA Prof. Dr. Rodolfo Rossi, La Plata, Provincia de Buenos Aires, Argentina}

Una mujer de 62 años, con antecedentes de hipotiroidismo, dislipidemia e hipertensión arterial en tratamiento, consultó por la presencia de una dermatosis pruriginosa en ambas piernas, de 7 meses de evolución. Examen físico dermatológico: presentaba en las caras anterior y lateral de los miembros inferiores placas eritemato-anaranjadas, algunas de límites netos, otras de límites difusos, con petequias en su superficie (Fotos 1, 2 y 3).

\section{1) ¿Cuál es su diagnóstico más probable?}

A. Púrpura por alteraciones hematológicas. B. Vasculitis cutánea de vasos pequeños. C. Dermatosis purpúrica pigmentaria. D. Dermatitis de contacto alérgica. E. Dermatitis por estasis.

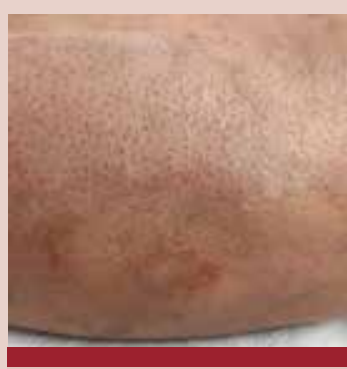

FOTO 1: Placas eritemato-anaranjadas en la pierna izquierda.

2) Los hallazgos que descartan esta entidad son:

A. Inflamación de células endoteliales con extravasación de glóbulos rojosy macrófagos que contienen hemosiderina. B. Alteración del tiempo de sangría o del coagulograma.

C. Vasculitis leucocitoclástica.

D. Eco-Doppler venoso de los miembros inferiores alterado. E. Las opciones B. y C. son correctas.

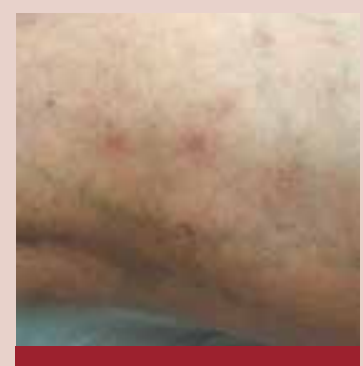

F0T02: Lesiones petequiales puntiformes.

3) ¿Qué enunciado considera verdadero basado en el cuadro de sospecha?

A. La mayoría son crónicas y no asocian compromiso sistémico. B. El compromiso cutáneo puede ser único o estar asociado a compromiso de órganos internos.

C. Son más frecuentes en los miembros inferiores y pueden producir prurito de intensidad variable.

D. Las respuestas A. y C. son correctas. E. Las respuestas B. yC. son correctas.

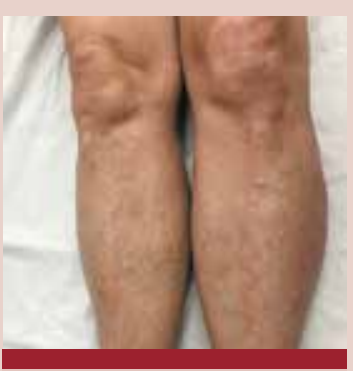

FOTO 3: Lesiones localizadas en la cara anterior de ambas piernas.

4) ¿Qué tratamiento indicaría en esta paciente según su diagnóstico presuntivo?

A. Transfusión de hemoderivados. B. Corticosteroides tópicos + antihistamínicos orales.

C. Corticosteroides sistémicos. D. Corticosteroides sistémicos + inmunosupresores.

E. Todas las opciones son correctas. La solución en la página 48 
y por su casa pasa todo tipo de personas que se acercan a pedirle dinero. Como ve desaparecer su fortuna y los que lo adoraban lo abandonan, se retira a una caverna y, al cavar en busca de raíces con las cuales alimentarse, encuentra un tesoro. Se lo da a Alcibíades, que también detestaba a los atenienses por haber prescindido de sus servicios, quien lo utiliza para pagar a los soldados destinados a destruir la ciudad y a prostitutas para que contagien sexualmente a la juventud de Atenas. Lo sorprendente de esta obra, que satiriza la ingratitud humana y las costumbres de la época, es el uso de distintas expresiones relacionadas con enfermedades venéreas como lepra blanco-grisácea (hoar leprosy), así llamada porque formaba costras blanquecinas en la piel, donde hoar (ho:) es homófono de whore (ho:), "puta", lo que al aceptar la versión inglesa correspondería a "lepra de putas".

\section{BIBLIOGRAFÍA}

Shakespeare W. Junto a los muros de Atenas. Acto IV, Escena 1. En: Timón de Atenas. Disponible en: 131615.pdf- http://biblioteca.org. ar/libros/131615.pdf

\section{DERMATÓLOGOS JÓVENES}

\section{Elección múltiple. LESIONES PURPÚRICAS EN LOS MIEMBROS INFERIORES / Respuestas}

\section{Respuestas correctas: 1) ( ; 2) E; 3) D; 4) B}

Las dermatosis purpúricas pigmentarias (DPP) son un grupo de lesiones cutáneas crónicas y recurrentes. Se caracterizan por petequias y/o púrpura y máculas marrón-anaranjadas pigmentarias que se localizan principalmente en las extremidades inferiores

Se clasifican en seis grupos según el patrón clínico: máculas purpúricas de color marrón rojizo 0 petequias del tamaño de una cabeza de alfiler en la enfermedad de Schamberg (DPP progresiva); parches o placas anulares con telangiectasias y petequias en los bordes en la púrpura anular telangiectoide (enfermedad de Majocchi); parches o placas liquenoides de color marrón dorado en el liquen aureus; pápulas y placas purpúricas liquenoides poligonales o redondas en la dermatosis liquenoide purpúrica pigmentada de Gougerot y Blum; máculas, pápulas o placas purpúricas escamosas y pruriginosas en la púrpura pruriginosa; máculas y parches purpúricos eccematosos en la púrpura de tipo eccematoide de Doucas-Kapetanakis (púrpura de tipo eccematoide). Aunque estos patrones representan las diferentes características morfológicas, la histopatología, el tratamiento y el pronóstico de todas las entidades son similares.

Se desconoce la causa de las DPP, pero se ha sugerido que varias situaciones comórbidas o medicamentos pueden estar asociados, como insuficiencia venosa crónica, diabetes mellitus, hiperlipidemia, fragilidad capilar e hipersensibilidad a fármacos ( $\mathrm{pa}$ racetamol, aspirina, antiinflamatorios no esteroides, diuréticos, dipiridamol, bezafibrato, etc.). Otras causas incluyen alergia de contacto a metales o colorantes, ropa e ingesta de alcohol.

El diagnóstico suele hacerse clínicamente, aunque en algunas circunstancias es necesaria la histopatología para diferenciarlo de vasculitis u otras enfermedades inflamatorias. Los hallazgos histológicos varían desde un infiltrado inflamatorio linfocítico perivascular, extravasación de eritrocitos y depósito de hemosiderina focal, hasta la infiltración liquenoide densa, espongiosis o infiltración granulomatosa.

La enfermedad tiene un curso benigno, aunque las DPP son cuadros crónicos, con exacerbaciones recurrentes. En términos de tratamiento, ninguna terapia única o combinada es superior a otra. Los tratamientos destinados a mejorar los factores etiológicos relacionados (p. ej., insuficiencia venosa crónica) pueden ayudar en algunos casos. También se recomienda la aplicación de emolientes y humectantes.

Basados en series y publicaciones de casos, los tratamientos utilizados con resultados terapéuticos satisfactorios son: en lo que respecta al tratamiento tópico, el uso de esteroides locales para reducir el prurito, 0 inhibidores de la calcineurina. Entre los tratamientos sistémicos, los antihistamínicos orales también se han utilizado como una medida de apoyo para controlar el prurito, se encuentra la terapia con flavonoides y altas dosis de ácido ascórbico (su mecanismo de acción está relacionado con una mejora de las funciones de la barrera endotelial vascular, a través de un aumento en la síntesis de colágeno). Otro agente que ocasionalmente ha mostrado resultados prometedores es la pentoxifilina. Por último, los informes que indican un efecto beneficioso de la fototerapia en el tratamiento de las DPP están presentes en la literatura médica.

\section{BIBLIOGRAFÍA}

- Kim DH, Seo SH, Ahn HH, Kye YC, et ál. Characteristics and clinical manifestations of pigmented purpuric dermatosis. Ann Dermatol 2015;27:404-410.

- Huang Y-K, Lin C-K, Wu Y-H. The pathological spectrum and clinical correlation of pigmented purpuric dermatosis- A restrospective review of 107 cases. J Cutan Pathol 2018;45:325-332.

- Plachouri K-M, Florou V, Georgiou S. Therapeutic strategies for pigmented purpuric dermatoses: a systematic literature review. J Dermatolog Treat 2019;30:105-109. 